\title{
Management of placenta accreta
}

Loïc Sentilhes ${ }^{1}$, M.D., Ph.D., François Goffinet ${ }^{2}$, M.D., Ph.D., Gilles Kayem ${ }^{3}$, M.D., Ph.D.

1. Department of Obstetrics and Gynecology, Angers University Hospital, Angers,

2. Department of Obstetrics and Gynecology, Maternité Port-Royal Hospital, Cochin APHP, University René Descartes, Paris,

3. Department of Obstetrics and Gynecology, Louis Mourier Hospital, APHP, University Paris-Diderot, Colombes, France.

\section{Corresponding author and reprint requests to:}

Loïc Sentilhes, M.D., PhD.

Department of Obstetrics and Gynecology

Angers University Hospital,

4, rue Larrey, 49000 Angers, France.

E-Mail: loicsentilhes@hotmail.com Tel: (33) $241357744 \quad$ Fax: (33) 241355789

Running title: Management of placenta accreta

\section{Disclosure of interests}

The authors have no direct or indirect commercial financial incentive associated with publishing the article. 


\section{Abbreviations}

ICU, intensive care unit

NICU, neonatal intensive care unit

$\mathrm{PPH}$, postpartum hemorrhage

\section{Key-message:}

Cesarean hysterectomy is the gold standard treatment for placenta accreta. For placenta percreta with bladder invasion or for young women who want the option of future pregnancy and who agree to close follow-up monitoring, conservative treatment is a valid option. 


\begin{abstract}
:
Cesarean-hysterectomy is considered the gold standard treatment for placenta accreta. In young women who want the option of future pregnancy and agree to close follow-up monitoring, conservative treatment is a valid option. Several key points of both cesarean hysterectomy and conservative treatment remain debatable, such as timing of delivery, attempted removal of the placenta, use of temporal internal iliac occlusion balloon catheters, ureteral stents, prophylactic embolization, and methotrexate. In cases of placenta percreta with bladder involvement, conservative treatment may be the optimal management. Regardless of the chosen option, the woman and her partner should be warned of the high risk of maternal complications related to an abnormally invasive placenta.
\end{abstract}

Key words: abnormally invasive placenta, placenta accrete or percreta, management, antenatal diagnosis, cesarean hysterectomy, conservative treatment. 


\section{Introduction}

Abnormally invasive placentas are a life-threatening condition characterized by placental villi being abnormally adherent to the myometrium due to the absence of, or defects in, the normal decidual basalis and the fibrinous Nitabuch's layer (1). They are commonly classified into three distinct grades according the degree of the placental villi invasion into the myometrium: placenta accreta (placental villi are attached to the decidual surface of the myometrium), placenta increta (placental villi more deeply invading into the myometrium) and placenta percreta (placental villi invade through the myometrium and the uterine serosa and sometimes into adjacent organs, such as the bladder) (2). In a clinical setting and daily practice, these histopathological differences are probably non-existent. Therefore, in this article, the term "placenta accreta" is used as a general term to describe all of these three conditions whereas the term percreta refers to placenta percreta (2).

Placenta accreta has become an important cause of maternal morbidity and mortality. For example, more than half of women are transfused or admitted to an intensive care unit (ICU) (3-4) and mortality rates as high as $7 \%$ have been reported to be associated with placenta percreta (5). Moreover, placenta accreta is the leading cause of peripartum hysterectomy (6), failed vessel ligation (7) and pelvic arterial embolization (8). Nevertheless, the optimal management of placenta accreta remains a topic of debate. The aim of our review was to assess the available useful procedures in an attempt to contribute to decreasing the morbidity of this condition.

\section{Methods}

Data for this review were identified based on searches of Medline, Current Contents, and PubMed from 1965 to January 2012, and references from relevant articles using the following 
search terms: "placenta accreta", "placenta increta", "placenta percreta", “morbidly adherent placenta", "antenatal diagnosis", “extirpative approach”, “cesarean-hysterectomy”, "conservative treatment". Only articles published in English were included. Further articles were identified by cross-referencing. The majority of publications on placenta accreta are cohort or case-control studies, or case series/reports and descriptive studies. No randomized controlled trials were retrieved. Articles on more general topics were selected on the basis of their relevance or interest as regards this specific subject with particular attention given to articles that dealt with the management of placenta accreta.

\section{Organization of the delivery}

\section{Role of prenatal identification of placenta accreta}

Prenatal identification of placenta accreta is essential in order to manage optimal delivery circumstances for these patients (9). The key role of this in the management of patients with placenta accreta was highlighted by Warshak et al. (4). These authors reported that patients with a pre-delivery diagnosis of placenta accreta for whom a cesarean hysterectomy with no attempted removal of the placenta was planned at 34-35 weeks gestation, required fewer units of packed red blood cells and tended to have a lower estimated blood loss than those with no pre-delivery diagnosis (4). This decrease in maternal morbidity for patients with a predelivery diagnosis of placenta accreta has also been reported by others (10).

Another advantage of prenatal identification is the possibility to plan the delivery in a suitable center with a multidisciplinary team and adequate equipment and resources including a maternity-oriented intensive care unit (ICU), an embolization unit with interventional radiologists, a blood bank capable of managing massive transfusion requirements, and the availability of other technical skills (urologists, vascular surgeons). It makes intuitive sense 
that expertise and experience are useful when managing difficult and somewhat uncommon problems. This has been demonstrated by Eller et al. who found that maternal morbidity is reduced in women with placenta accreta who delivered in a tertiary care hospital with a multidisciplinary care team in comparison with those managed in standard obstetric care facilities (11).

\section{Timing of delivery}

The optimal timing of delivery for the patients with placenta accreta remains controversial. Whatever the gestational age chosen, practitioners have to plan an organization to include the unexpected occurrence of possible cases of acute bleeding requiring an emergency cesarean section. In a large multicenter trial, $23.9 \%$ (27/113) of women with placenta accreta in whom cesarean section was planned had an emergency delivery (8). O'Brien et al. reported that after 35 weeks, $93 \%$ of patients with placenta accreta experienced hemorrhage necessitating delivery (5). In order to decrease this risk of emergency delivery, Warshak et al. proposed that patients with a highly suspected placenta accrete should be delivered by cesarean hysterectomy at 34-35 weeks (4). Compared with patients with no pre-delivery diagnosis of placenta accreta ( $\mathrm{n}=37$; mean gestation at delivery $33.9 \pm 1.1$ weeks), those with a pre-delivery diagnosis ( $n=62$; mean gestation at delivery $34.7 \pm 1.2$ weeks $)$ demonstrated significantly higher rates of admission to the neonatal ICU (86\% compared with 60\%), and longer neonatal hospital stays (10.7 \pm 1.9 compared with $6.9 \pm 2.1$ days). Despite the limited size of their patient cohort, this study suggested that higher neonatal morbidity may occur if the cesarean is planned before 36 weeks of gestation (4). Finally, an analytical decision model showed that the preferred gestational age at which to deliver these individuals at high risk is almost certainly at or beyond 34 weeks, but not later than 37 weeks (12). Nevertheless, it has been emphasized that the current literature detailing the probability of events related to emergency 
delivery of women with suspected placenta accrete, is not definitive enough to allow the unequivocal establishment of a single ideal gestational age at which to deliver the women who are at high risk (12). Therefore, in absence of any bleeding, it seems currently reasonable to plan delivery at about 35-36 weeks gestation in cases of suspected placenta accreta. This opinion seems to be shared by the majority of American practitioners as about $50 \%$ of respondents reported in two national surveys to have planned the cesarean section at 36 weeks gestation $(13,14)$.

\section{Procedures}

There are four basic options for management of placenta accreta: the extirpative method, the cesarean hysterectomy, conservative treatment and the one-step conservative surgery which is an alternative conservative technique. Whatever the option chosen, when placenta accreta is suspected before delivery in a woman with an anterior placenta previa, it is recommended to perform a vertical fundal uterine incision to avoid the placenta and reduce the risk of massive postpartum hemorrhage (PPH).

\section{The extirpative method}

This procedure consists of performing a forcible manual removal of the placenta delivery in an attempt to obtain an empty uterus. The technique is associated with a higher rate of massive PPH and subsequent peripartum hysterectomy than the conservative approach (1516). Therefore, this option should be abandoned when other procedures are available (17). Unfortunately, this happens in most cases with undiagnosed placenta accrete, i.e. it is not a voluntary decision but rather the result of an unforeseen intraoperative complication when an accreta is not suspected. That concept underlines the importance of preoperative diagnosis. 


\section{Cesarean section hysterectomy}

This procedure consists of performing a hysterectomy after the birth of the child without attempting removal of the placenta when placenta accreta is strongly suspected antenatally or after an attempted placental removal when the diagnosis of placenta accreta is not made until during delivery. This option is currently recommended by the American College of Obstetrics and Gynecology as well as various authors, and is considered the gold standard treatment for placenta accreta $(1,5,18,19,20)$. The maternal mortality rate of cesarean hysterectomy seems to be relatively low. Among 95 deaths after 1461270 births between 2000 and 2006, only one death secondary to placenta accreta had been reported in the United States (21). Nevertheless, mortality rates as high as $7 \%$ have been reported to be associated with placenta percreta (5). Surprisingly, there are very few case series which have assessed maternal morbidity after cesarean hysterectomy. The most methodologically sound studies have only recently been published $(3,4,22,23)$. Table 1 summarizes maternal morbidity of the largest reported retrospective case series of a peripartum hysterectomy for placenta accreta. One of the most interesting is the study of Eller et al. (3), who found, in a series of 76 cases of cesarean hysterectomy for placenta accreta a $42.1 \%$ transfusion rate ( $\geq 4$ red blood cells), $28.9 \%$ cystotomy rate, ureteral injury in $6.6 \%$ of the women, while infectious complications occurred in $33.3 \%$ and in all major morbidity among $59 \%$ of the women (Table 1). In spite of some methodological flaws (24), it appears that maternal morbidity is significantly reduced when no attempt is performed to remove the placenta (3). Warshak et al. (4) described morbidity of cesarean hysterectomy for placenta accreta at a similar level to what Eller et al. (3) observed. In that series of 62 cases of cesarean-hysterectomy, performed without attempting to remove the placenta, the average estimated blood loss, number of red blood cells transfused, and operative time was $2344 \mathrm{~mL}, 4.7$ and 194 minutes, respectively, while 
the rates of bladder injuries, ureteral injuries and neonatal ICU referrals were $23 \%, 8 \%$ and $72 \%$, respectively (4) (Table 1).

The main advantage of cesarean hysterectomy (without attempted removal of the placenta) is probably to limit the risk of bleeding in order to reduce maternal morbidity, although this still remains high $(3,25)$. Its major drawback is the loss of fertility, which may seem particularly unwarranted when placenta accreta is not confirmed histologically (this was the case in $28 \%$ of cases of prenatal suspicion of placenta accreta in the study of Eller et al. (3)). Moreover, there is a lack of consensus regarding several detailed key points of the cesarean hysterectomy strategy:

- Should preventive arterial embolization be performed after birth?

This has been proposed to decrease maternal morbidity following cesarean hysterectomy. Nevertheless, only one limited study including a small cohort $(n=26)$ has addressed this issue. Angtsmann et al. observed a significant decrease in blood loss, number of transfused patients and number of red blood cell units transfused in a short ambispective cohort of women who underwent arterial embolization before hysterectomy compared with patients who had a hysterectomy just after the birth of the child (21). In our practice, we do not use prophylactic embolization before performing an hysterectomy.

- Should temporal internal iliac occlusion balloon catheters be used?

The main potential advantage of these catheters is to reduce blood loss and its inherent morbidity by reducing the blood flow distal to the site of occlusion, through decreasing the blood flow to the uterine arteries after a balloon inflation in the internal iliac arteries, as well as improving the visualization of the surgical field until hemostasis or hysterectomy can be achieved. Nevertheless, some authors have advocated that routine inflation of the balloons immediately following delivery of the infant may exacerbate collateral blood flow (26). It has been suggested that the extensive network of collateral vessels present in the gravid pelvis 
may explain some failures of occlusive balloons, whereas collateral circulation from cervical, ovarian, rectal, femoral, lumbar, and sacral arteries may also contribute to the overall blood loss (26).

Another advantage of the preoperative placement of hypogastric catheters is that they can be used for postoperative embolization should bleeding complications occur after an intended hysterectomy. However, owing to differences regarding the technique, protocols, heterogeneous sets of management and only small cases series with discordant results $(26,27,28,29)$, there is currently no consensus regarding their use. In particular, the Royal College of Obstetricians and Gynaecologists has underlined the need of further evaluation before its recommendation (30). Among the only three retrospective comparative studies that have assessed maternal outcome following cesarean hysterectomy alone vs. placement of balloon catheters before performing cesarean hysterectomy $(26,27,28)$, there were no differences for estimated blood loss, transfused blood products, operative time and length of hospitalization between patients who had balloon catheters plus hysterectomy $(n=19)$ and those who had hysterectomy alone in the largest series $(n=50)(26)$. As this procedure is associated with a high rate of harmful effects, it seems difficult to recommend this controversial procedure requiring fetal radiation in the absence of a significant decrease of maternal morbidity. In fact, it has been estimated that surgical complications, such as puncture site hematomas, false aneurysms, dissection of femoral arteries, air in pressurized lines, symptomatic hypotension, and bowel or ureteral injury, and serious thromboembolic events, occur approximately in 6-16\% and about $5 \%$ of women, respectively (29).

- Should ureteral stents be used?

There is no study that has specifically assessed this issue. The most interesting findings are provided by the study of Eller et al. (3). In this study, in cases of suspected placenta accreta, a preoperative ureteral stent placement was attempted in 44\% of cases $(25 / 57)$. The stent 
placement was achieved on the right and left sides in only $68 \%(17 / 25)$ of cases, on only one side in $16 \%(4 / 25)$ of cases, and neither side in $16 \%(4 / 25)$. Women with preoperative bilateral ureteral stents had a lower incidence of early morbidity compared with women without stents $(18 \%(3 / 17)$ versus 55\% (22/40), $\mathrm{p}=.018)$. Moreover, these women had a nonsignificant reduction in ureteral injury ( 0 vs. $7 \%$ ). Therefore, this study suggests that ureteral stent placement may help to reduce the risk of ureteral injury (3). However, further evaluation is required before considering routine ureteral stents used for all women with placenta accreta. In recent American surveys, $26 \%$ to $35 \%$ of respondents reported using ureteral stents in planning for a placenta accreta case $(13,14)$.

\section{Conservative treatment}

This option consists of delivering the child, tie and then cut the umbilical cord at its base to leave the placenta in place adhering either partially or totally to the myometrium, and to close the hysterotomy (Fig. 1). Conservative treatment can also be performed following a vaginal delivery when the placenta is not delivered and no plane of cleavage is found between the uterus and the placenta, but only if this attempt to remove the placenta was careful, without force or insistence. Conservative treatment avoids a hysterectomy in about $75-80 \%$ of cases but is associated with a risk of transfusion requirements, infection and severe maternal morbidity $(15,16,31,32)$. In addition, it requires long-term monitoring. Until recently, only a very limited amount of data regarding maternal outcome after conservative management was available, based only on case reports and short case series from individual tertiary-care institutions $(15,16,32)$.

In order to obtain statistical power and satisfactory external validity, a French multicenter retrospective study was conducted to determine the maternal outcome after conservative treatment (31). The results of this study concerning 167 cases of placenta accreta (59\% of 
placentas were partially left in place and $41 \%$ were totally left in place) are summarized in Table 2 (31). They confirm the overall success rate previously published $(15,16)$ with uterine preservation (no hysterectomy) in $78.4 \%$ of cases, and severe maternal morbidity in $6 \%$ (10/167) (31). One maternal death related to multiorgan failure occurred in a patient with medullary aplasia, nephrotoxicity with acute renal failure, followed by peritonitis with septic shock, after the injection of methotrexate in the umbilical cord. An empty uterus was obtained spontaneously in $75 \%$ of cases after a median of 13.5 weeks (min: 4 weeks, max: 60 weeks). Hysteroscopic resection and/or curettage were performed to obtain an empty uterus in 25\% with a median of 20 weeks (min: 2 weeks, max: 45 weeks) (31). In a recent American survey, $32 \%$ of providers had attempted conservative management for placenta accreta (13).

Nevertheless, similarly to the cesarean hysterectomy, the precise modalities of conservative treatment remain to be determined:

- Should a routine gentle attempted removal of the placenta be made in cases of suspected prenatal placenta accreta?

The main advantage is not only to avoid leaving an in situ placenta, in case of false-positive imaging, but also to be able to remove the "non-accreta" portion when the placenta adheres partially to the myometrium, in order to reduce the volume left in the uterus. However, this attempted removal of the placenta can cause severe bleeding with the risk of maternal complications and hysterectomy. Our current practice is to attempt gently to remove the placenta only in cases of unconvincing findings of placenta accreta. An example was a nulliparous woman with a history of curettage in whom the ultrasonography revealed only intraplacental lacunae in a low-lying anterior placenta, with inconclusive findings on magnetic resonance imaging, as well as no evidence of placenta accreta during the cesarean.

- Should methotrexate adjuvant treatment be administered? 
Some authors have proposed the use of methotrexate to hasten the placental resolution (33). Its efficacy for this indication has never been demonstrated and only case reports and very short case series with no control group have been reported (34). The RCOG does not recommend its routine use (30). The low rate of placental cell turnover compared to what is observed in early pregnancy suggests a much lower efficacy of methotrexate in late compared to early pregnancy. In addition, methotrexate exposes the patient to the risk of neutropenia or medullary aplasia, even with a single dose in a young patient for the treatment of ectopic pregnancy (35). These types of side-effects can have a dramatic impact in a patient with an intrauterine placenta with a $30 \%$ risk of infectious complications (31). Finally, the only case of maternal death after conservative treatment was secondary to a cascade of complications (bone marrow suppression, sepsis, renal failure) attributed to an intra-umbilical cord administration of methotrexate (31). For these reasons, we do not advocate the use of methotrexate in cases of conservative treatment.

- Should a preventive uterine devascularization be carried out in the absence of bleeding? We have only very limited data to answer this question. This preventive devascularization can be achieved by the usual techniques of PPH management (embolization, bilateral uterine artery ligation, stepwise uterine devascularization, bilateral ligation of hypogastric arteries), although these uterine-sparing procedures seem to be less effective in cases of placenta accreta (7-8). As Angstmann et al. have shown that the implementation of a preventive embolization before performing hysterectomy may reduce the risk of blood loss (22), it is possible to speculate that within the framework of conservative treatment a prophylactic devascularization could prevent the occurrence of secondary hemorrhage and reduce the risk of blood loss (36) (Fig. 2). It could also accelerate the placental resolution. In contrast, these devascularization techniques may have harmful effects $(8,31)$. In the French multicenter series of 167 placenta accrete cases treated conservatively, the only two cases of uterine 
necrosis occurred in two of 62 patients who underwent arterial embolization (31). However, further evaluation is required to answer this issue.

- How should patients with conservative management be monitored?

Unfortunately, there are no data available regarding this important issue. In our practice, we $u$ administer prophylactic antibiotics during five days and discharge the patient from hospital on the $8^{\text {th }}$ postoperative day. Moreover, subsequent follow-up requires usually weekly visits until the complete resorption of the placenta. The visits include a clinical examination (bleeding, temperature, pelvic pain), pelvic ultrasound (volume of retained tissue) and laboratory tests for infection (hemoglobin and leukocytes count, C-reactive protein, vaginal sample for bacteriological analysis) (31).

\section{The one step-conservative surgery}

This alternative conservative procedure has been mainly described by one author $(37,38,39)$. It consists of resecting the invaded area together with the placenta and performing the reconstruction as a one-step procedure (38). The main stages of this alternative technique achieved through a median or transverse suprapubic incision are a) vascular disconnection of newly-formed vessels and the separation of invaded uterine from invaded vesical tissues; b) performing an upper-segmental hysterectomy; c) resection of all invaded tissue and the entire placenta in one piece with previous pervious local vascular control; d) use of surgical procedures for hemostasis; e) myometrial reconstruction in two planes and f) bladder repair if necessary (39). This procedure does not appear to alter the subsequent obstetrical outcome. Palacios-Jaraquemada has reported 45 pregnancies following a one step-procedure for placenta accreta. Of these, 44 were uneventful and only one was complicated by a recurrence of placenta accreta (38). 
As we have a limited experience with the one-step conservative surgery (40), we find it difficult to reach a definite opinion regarding this technique. Nevertheless, we feel that this procedure may be less reproducible than a conservative treatment, in particular because achieving hemostasis may be very challenging for an inexperienced team.

In summary, the available useful procedures in an attempt to decrease the morbidity of placenta accreta remain to be clearly determined by further studies. The prospective PACCRETA study has been launched in order to answer some of the questions raised in this article (9).

Finally, we would like to emphasize the adverse effects related to false positive or negative findings with regard to a prenatal suspicion of placenta accreta using Doppler ultrasound and/or magnetic resonance imaging. These two imaging examinations do unfortunately not achieve a sufficiently high level of accuracy to detect a placenta accreta (9). The consequences of a false negative result are obvious, i.e. increased maternal morbidity (4, $9,10,11)$ ) and have been mentioned above. Similarly, caregivers should be aware that false positive results, which may occur in $28 \%$ of cases (3), also increase maternal morbidity. They may lead caregivers to perform unnecessary radical or conservative-surgical procedures and expose the women to their inherent complications. This includes in all cases a high level of anxiety and for the cesarean hysterectomy, loss of fertility and surgical injury; while for the conservative treatment, infection/sepsis as well as bleeding that may lead to a hysterectomy can result, to which can be added the long period of follow-up and anxiety. Caregivers should therefore pay particular attention to both false negative and positive of prenatal imaging examinations. 


\section{Cesarean hysterectomy or conservative treatment?}

There is only one small retrospective comparative study assessing the maternal outcome following cesarean hysterectomy $(n=16)$ compared to conservative treatment $(n=10)(41)$. No differences were observed between the two groups except for the estimated blood loss which was lower in the conservative treatment group $(3625 \mathrm{~mL} \pm 2154$ vs. $900 \mathrm{~mL} \pm 754)$ for a rate of uterine preservation of $60 \%(6 / 10)$ (41). Compared to cesarean hysterectomy, the main disadvantage of conservative treatment is the length of the management and the need for prolonged follow-up of several months, while the main benefit is the preservation of the uterus and thus fertility preservation. Fertility and subsequent obstetrical prognosis of patients who desire a pregnancy after conservative treatment does not appear to be altered (17). The main risk in case of future pregnancies seems to be the recurrence of placenta accreta estimated at $28.6 \%$ (95\% confidence interval $11.3-52.2 \%$ ) (17). Due to the small number of studies, a limited inclusion of patients in these studies, the inhomogeneity of patient characteristics and their management, and methodological flaws, it is very difficult to compare maternal morbidity after conservative treatment vs. cesarean hysterectomy. However, it is possible that severe maternal morbidity is increased in cases of conservative treatment because infectious complications, uterine necrosis and secondary hemorrhage associated to conservative treatment can be dramatic. Thus, it seems reasonable to propose in the present state of knowledge, a cesarean-hysterectomy if the patient has no desire for a future pregnancy, is at a relatively advanced reproductive age and is multiparous. Nevertheless, we believe that conservative management is an option for patients who are properly counseled and motivated, in particular, for women who want the option of a future pregnancy and who agree to close follow-up monitoring in centers with adequate equipment and resources $(24,25,31)$. However, regardless of the chosen option (cesarean hysterectomy 
or conservative treatment), the patient and her partner should be warned of the high risk of maternal complications related to placental disease.

\section{Placenta percreta with bladder invasion}

The bladder is the most frequently invaded adjacent organ when there is a placenta percreta

(Fig. 3). In this condition, maternal morbidity is severe and high, with mostly urological complications (72.2\%), including partial cystectomy (44\%), bladder lacerations (26\%), urinary fistula (13\%), gross hematuria (9\%), ureteral transaction $(6 \%)$ and later small capacity bladder (4\%). A maternal death occurred in $5.6 \%$ of cases (42). Pre-operative placement of a ureteral catheter is probably of great help to minimize ureteral complications (3). In this condition, it is likely that conservative treatment has a lower morbidity compared to radical treatment $(25,31,43)$. This opinion was already shared by $69 \%$ of the members of the American Society of Perinatal Obstetricians in 1996 (5).

\section{Discussion}

In the case of a prenatal suspected placenta accreta, the extirpative method must now be abandoned. It seems reasonable to plan delivery at approximately 35-36 weeks of gestation. The advantages and disadvantages of cesarean hysterectomy and conservative management should be clearly exposed to the patient and the partner, who must be involved in deciding on the chosen option. Considering the present state of knowledge, it seems reasonable to propose a cesarean hysterectomy if the patient has no desire for a future pregnancy, is at a higher reproductive age and is parous. In these latter cases, preoperative ureteral stent placement as well as prophylactic embolization before hysterectomy may be considered in order to reduce maternal morbidity, whereas the benefit-harm balance is against the routine use of internal iliac occlusion balloon catheters. In contrast, if the patient wants the possibility of another 
pregnancy, is young and nulliparous, conservative treatment with no adjuvant methotrexate therapy should be proposed. In rare cases of placenta percreta with bladder invasion, it seems reasonable to focus on conservative treatment. 


\section{Acknowledgement}

The authors are grateful to Richard Medeiros, Rouen University Hospital Medical Editor, for his valuable editorial assistance. 


\section{Funding}

The authors declare no source of funding 


\section{References}

1. Tseng JJ, Chou MM, Hsiehb YT, Wene MC, Hob ES, Hsuf SL. Differential expression of vascular endothelial growth factor, placenta growth factor and their receptors in placentae from pregnancies complicated by placenta accreta. Placenta 2006; 27:70-78.

2. Oyelese Y, Smulian JC. Placenta previa, placenta accreta, and vasa previa. Obstet Gynecol 2006;107:927-41.

3. Eller AG, Porter TF, Soisson P, Silver RM. Optimal management strategies for placenta accreta. BJOG 2009;116:648-54.

4. Warshak CR, Ramos GA, Eskander R, Benirschke K, Saenz C, Kelly TF, et al. Effect of predelivery diagnosis in 99 consecutive cases of placenta accreta. Obstet Gynecol 2010;115:65-9.

5. O'Brien JM, Barton JR, Donaldson ES. The management of placenta percreta: conservative and operative strategies. Am J Obstet Gynecol 1996;175:1632-7.

6. Daskalakis G, Anastasakis E, Papantoniou N, Mesogitis S, Theodora M, Antsaklis A. Emergency obstetric hysterectomy. Acta Obstet Gynecol Scand 2007;86:223-227.

7. Sentilhes L, Trichot C, Resch B, Sergent F, Roman H, Marpeau L, et al. Fertility and pregnancy outcomes following uterine devascularization for severe postpartum haemorrhage. Hum Reprod 2008;23:1087-92.

8. Sentilhes L, Gromez A, Clavier E, Resch B, Verspyck E, Marpeau L. Predictors of failed pelvic arterial embolization for severe postpartum hemorrhage. Obstet Gynecol 2009;113:992-9.

9. Kayem G, Deneux-Tharaux C, Sentilhes L; PACCRETA group. PACCRETA: clinical situations at high risk of placenta ACCRETA/percreta: impact of diagnostic methods and management on maternal morbidity. Acta Obstet Gynecol Scand 2013;92:476-82. 
10. Tikkanen M, Paavonen J, Loukovaara M, Stefanovic V. Antenatal diagnosis of placenta accreta leads to reduced blood loss. Acta Obstet Gynecol Scand 2011;90:1140-6.

11. Eller AG, Bennett MA, Sharshiner M, Masheter C, Soisson AP, Dodson M, et al. Maternal morbidity in cases of placenta accreta managed by a multidisciplinary care team compared with standard obstetric care. Obstet Gynecol 2011;117:311-7.

12. Robinson BK, Grobman WA. Effectiveness of timing strategies for delivery of individuals with placenta previa and accreta. Obstet Gynecol 2010;116:835-42.

13. Esakoff TF, Handler SJ, Granados JM, Caughey AB. PAMUS: placenta accreta management across the United States. J Matern Fetal Neonatal Med 2012;25:761-5.

14. Jolley JA, Nageotte MP, Wing DA, Shrivastava VK. Management of placenta accreta: a survey of Maternal-Fetal Medicine practitioners. J Matern Fetal Neonatal Med 2012;25:756-60.

15. Kayem G, Davy C, Goffinet F, Thomas C, Clement D, Cabrol D. Conservative versus extirpative management in cases of placenta accreta. Obstet Gynecol 2004;104:531-6.

16. Bretelle F, Courbiere B, Mazouni C, Agostini A, Cravello L, Boubli L et al. Management of placenta accreta: morbidity and outcome. Eur J Obstet Gynecol Reprod Biol 2007;133:34-9.

17. Sentilhes L, Kayem G, Ambroselli C, Provansal M, Fernandez H, Perrotin F, et al. Fertility and pregnancy outcomes following conservative treatment for placenta accreta. Hum Reprod 2010;25:2803-10.

18. Oyelese Y, Smulian JC. Placenta previa, placenta accreta, and vasa previa. Obstet Gynecol 2006;107:927-41.

19. American College of Obstetricians and Gynecologists. Postpartum hemorrhage. ACOG Educational Bulletin 243. Washington, DC: ACOG; 1998. 
20. Publications Committee, Society for Maternal-Fetal Medicine, Belfort MA. Placenta accreta. Am J Obstet Gynecol. 2010 Nov;203:430-9.

21. Clark SL, Belfort MA, Dildy GA, Herbst MA, Meyers JA, Hankins GD. Maternal death in the 21 st century: causes, prevention, and relationship to cesarean delivery. Am J Obstet Gynecol 2008;199:36.e1-36.e5.

22. Angstmann T, Gard G, Harrington T, Ward E, Thomson A, Giles W. Surgical management of placenta accreta: a cohort series and suggested approach. Am J Obstet Gynecol 2010;202:38.e1-9.

23. Hoffman MS, Karlnoski RA, Mangar D, Whiteman VE, Zweibel BR, Lockhart JL, Camporesi EM. Morbidity associated with non emergent hysterectomy for placenta accreta. Am J Obstet Gynecol 2010;202:628.e1-5.

24. Kayem G, Sentilhes L, Deneux-Tharaux C. Management of placenta accreta. BJOG 2009;116:1536-7.

25. Sentilhes L, Descamps P, Goffinet F. Arteriovenous malformation following conservative treatment of placenta percreta with uterine artery embolization but no adjunctive therapy. Am J Obstet Gynecol 2011;205:e13.

26. Shrivastava V, Nageotte M, Major C, Haydon M, Wing D. Case-control comparison of cesarean hysterectomy with and without prophylactic placement of intravascular balloon catheters for placenta accreta. Am J Obstet Gynecol. 2007;197:402.e1-5.

27. Levine AB, Kuhlman K, Bonn J. Placenta accreta: comparison of cases managed with and without pelvic artery balloon catheters. J Matern Fetal Med 1999;8:173-6.

28. Bodner LJ, Nosher JL, Gribbin C, Siegel RL, Beale S, Scorza W. Balloon-assisted occlusion of the internal iliac arteries in patients with placenta accreta/percreta. Cardiovasc Intervent Radiol 2006;29:354-61. 
29. Dilauro MD, Dason S, Athreya S. Prophylactic balloon occlusion of internal iliac arteries in women with placenta accreta: Literature review and analysis. Clin Radiol 2012;67:51520

30. Royal College of Obstetricians and Gynaecologists. Green-top Guideline No. 27: Placenta praevia, placenta praevia accrete and vasa praevia: diagnosis and management. London: RCOG; 2011.

31. Sentilhes L, Ambroselli C, Kayem G, Provansal M, Fernandez H, Perrotin F, et al. Maternal outcome after conservative treatment of placenta accreta. Obstet Gynecol 2010; 115: 526-34.

32. Alanis M, Hurst BS, Marshburn PB, Matthews ML. Conservative management of placenta increta with selective arterial embolization preserves future fertility and results in a favorable outcome in subsequent pregnancies. Fertil Steril 2006;86:1514.e3-7.

33. Mussalli GM, Shah J, Berck DJ, Elimian A, Tejani N, Manning FA. Placenta accreta and methotrexate therapy: three case reports. J Perinatol 2000;20:331-4.

34. Timmermans S, van Hof AC, Duvekot JJ. Conservative management of abnormally invasive placentation. Obstet Gynecol Surv 2007;62:529-39.

35. Isaacs Jr. JD, McGehee RP, Cowan BD. Life-threatening neutropenia following methotrexate treatment of ectopic pregnancy: a report of two cases. Obstet Gynecol 1996;88:694-6.

36. Bouvier A, Sentilhes L, Thouveny F, Bouet PE, Gillard P, Willoteaux S, Aubé C. Planned caesarean in the interventional radiology cath lab to enable immediate uterine artery embolization for the conservative treatment of placenta accreta. Clin Radiol 2012;67:1089-94. 
37. Palacios-Jaraquemada JM, Pesaresi M, Nassif JC, Hermosid S. Anterior placenta percreta: surgical approach, hemostasis and uterine repair. Acta Obstet Gynecol Scand 2004;83:738-44.

38. Palacios-Jaraquemada JM. Diagnosis and management of placenta accreta. Best Pract Res Clin Obstet Gynecol 2008;22:1133-48.

39. Palacios-Jaraquemada JM. Placental adhesive disorders. 2012 Walter de Gruyter GmbH and Co. KG, Berlin/Boston.

40. Kayem G, Deis S, Estrade S, Haddad B. Conservative management of a near-term cervico-isthmic pregnancy, followed by a successful subsequent pregnancy: a case report. Fertil Steril 2008;89:1826.e13-5

41. Amsalem H, Kingdom JC, Farine D, Allen L, Yinon Y, D'Souza DL, et al. J Obstet Gynaecol Can 2011;33:1005-10.

42. Washecka R, Behling A. Urologic complications of placenta percreta invading the urinary bladder: a case report and review of the literature. Hawaii Med J 2002; 61: 66-69.

43. Hayes E, Ayida G, Crocker A. The morbidly adherent placenta: diagnosis and management options. Curr Opin Obstet Gynecol 2011;23:448-53. 


\section{Legends for Figures}

Fig. 1. Peri-operative view of a placenta percreta left in situ after the closing of the vertical fundal uterine incision.

Fig. 2. Angiograms respectively before and after a prophylactic pelvic arterial embolization for placenta percreta of the left ( $a$ and $b$ ) and right ( $c$ and $d$ ) sides. Note the significant decrease of the vascularization at the end of the procedure.

Fig. 3. Hysteroscopic view of a placenta percreta with a bladder invasion. 